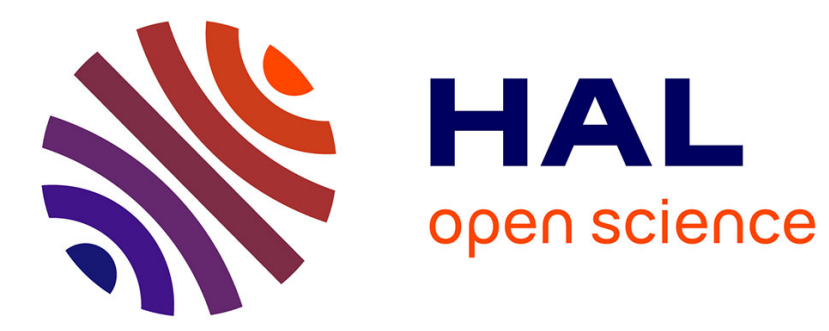

\title{
Premixed flames in large scale and high intensity turbulent flow
}

\author{
P. Clavin, G. Joulin
}

\section{To cite this version:}

P. Clavin, G. Joulin. Premixed flames in large scale and high intensity turbulent flow. Journal de Physique Lettres, 1983, 44 (1), pp.1-12. 10.1051/jphyslet:019830044010100 . jpa-00232137

\section{HAL Id: jpa-00232137 https://hal.science/jpa-00232137}

Submitted on 1 Jan 1983

HAL is a multi-disciplinary open access archive for the deposit and dissemination of scientific research documents, whether they are published or not. The documents may come from teaching and research institutions in France or abroad, or from public or private research centers.
L'archive ouverte pluridisciplinaire HAL, est destinée au dépôt et à la diffusion de documents scientifiques de niveau recherche, publiés ou non, émanant des établissements d'enseignement et de recherche français ou étrangers, des laboratoires publics ou privés. 


\title{
LE JOURNAL DE PHYSIQUE-LETTRES
}

J. Physique - LETTRES 44 (1983) L-1 - L-12

1'er JANVIER 1983, PAGE L-1

Classification

Physics Abstracts

47.70

\section{Premixed flames in large scale and high intensity turbulent flow}

\author{
P. Clavin and G. Joulin $\left(^{*}\right)$ \\ Laboratoire de Dynamique et Thermophysique des Fluides (**), \\ Université de Provence, Centre de St-Jérôme, 13397 Marseille Cedex 13, France
}

(Reçu le 11 mai 1982, révisé le ler octobre, accepté le 10 novembre 1982)

\begin{abstract}
Résumé. - La théorie récente de Clavin et Williams [1] sur les fronts de flammes prémelangées dans les écoulements inhomogènes et instationnaires est généralisée au cas où l'amplitude de déplacement $\mathrm{du}$ front est grande. Les effets convectifs associés à l'expansion du gaz sont pleinement pris en compte dans l'étude de la structure locale du front plissé. On montre qu'un seul scalaire mesurant l'étirement du front contrôle localement la forme et la dynamique de la flamme. Ce scalaire peut se décomposer en deux termes représentant respectivement la contribution de la géométrie de la flamme (courbure du front avançant avec une vitesse normale prescrite) et celle de l'inhomogénéité de l'écoulement caractérisée par le tenseur du taux des déformations.
\end{abstract}

\begin{abstract}
The recent theory of Clavin and Williams [1] concerning the premixed flame fronts in non uniform as well as unsteady flows is extended to the case of large amplitudes of the front corrugation. The convective effects associated with the gas expansion are fully taken into account in the study of the flame structure. Only one scalar " the flame stretch " is shown to control the shape and the motion of the front. This stretch can be split in two parts, one accounts for the geometry of the front (mean curvature) and the other for the non uniformity of the flow (rate of strain tensor).
\end{abstract}

1. Introduction. - When the flame thickness $d\left(\sim 10^{-2} \mathrm{~cm}\right.$ at the normal conditions) is smaller than the characteristic length scale $\Lambda$ of the upstream gas flow, $\varepsilon=d / \Lambda \ll 1$, the dynamical properties of a premixed flame are controlled by the coupling of two kinds of mechanisms which can be studied separately $[1,2]$ :

a) Inside the flame thickness the temperature increases from $T_{\mathrm{u}}$ to $T_{\mathrm{b}}$ and the convective and diffusive transport processes of mass and energy control the local structure of the wrinkled front. This structure links the flame speed to the shape of the front and to the local characteristics of the upstream gas flow.

b) But, in addition, hydrodynamical effects are developed upstream and downstream of the front. When the Mach number is sufficiently small, the density $\rho$ can be considered to be constant in the gas flow outside the flame thickness, but with a different value in the unburnt and burnt gases, $\rho_{\mathrm{u}} \neq \rho_{\mathrm{b}}$. This difference produces the deflection of the streamlines through the tilted front.

(*) Permanent address : Laboratoire d'énergétique et détonique, L.A. 193 du C.N.R.S., rue Guillaume-leTroubadour, 86034 Poitiers Cedex, France.

$\left({ }^{* *}\right)$ L.A. no 72 au C.N.R.S. 
According to the incompressible fluid mechanics, the flow is modified on a distance of the same order as the wrinkles of the front.

Thus, the hydrodynamical effects make the incoming flow depend on the motion and on the shape of the front, but, in turn, due to the flame structure, the flame motion depends on the local characteristics of the flow. The first study of this feedback mechanism was carried out forty years ago by Darrieus [3] in 1938 and by Landau [4] in 1944 but in a crude approximation : the structure of the wrinkled flame was assumed not to be modified from the steady planar case. This leads to a strong instability of the front : the flow produced by the wrinkling leads to a convective motion of the front which amplifies the initial wrinkles. But, clearly, the overlooked diffusive effects developed in the transverse dilation must modify this result at least for short wave-lengths. The first study of the wrinkled flame structure was carried out twenty years later by Barenblatt et al. [5] but in the simplifying approximation of the thermal-diffusive model where the convective effects produced by the difference between $\rho_{\mathrm{u}}$ and $\rho_{\mathrm{b}}$ were neglected. Much deeper insights have been provided by more recent analysis such as that presented in reference [6] in which the asymptotic analysis in large value of the activation energy [7] has been used. But it is only very recently that a complete analysis of the flame structure for small amplitudes of the front corrugation has been carried out [1] by using a multiscale analysis based on the disparity of the two scales $d$ and $\Lambda$. The convective effects neglected in [5] and in [6] are proved in [1] to be of the same order of magnitude as the diffusion effects but are always stabilizing. Thus, according to the results of Pelcé and Clavin [2] concerning the limits of stability of planar fronts, it turns out that the acceleration of gravity and the diffusive effects can overcome the hydrodynamical instability mechanism of Darrieus [3] and Landau [4] provided that the flame propagates downwards with a slow enough speed.

We present in this paper a natural extension of the study [1] of the wrinkled flame structure to the nonlinear case for large amplitudes of the front corrugation. But the dimension of the wrinkles is also assumed to be large compared to the flame thickness $d$ and, as in [1], $\varepsilon=d / \Lambda \ll 1$ is used as a perturbation parameter of the analysis. Furthermore, the asymptotic expansion in large values of the reduced activation is used as in [1] and [6]. The analysis does not involve any assumption concerning the magnitude of the gas expansion, thus, the convective effects produced by the deflection of the streamlines are properly taken into account in the flame structure. The nonlinear equation for the local evolution of the front is obtained in terms of the upstream gas flow as it is modified by the flame. The modification of the flow by the hydrodynamical effects described in $b$ ) can be analytically investigated in the linear approximation [2]. But, in the general case, the corresponding fluid mechanical problem is non linear and is left to be one of the most difficult in flame theory. This problem is not addressed here where only the local structure of the flame is investigated for turbulent flows of high intensity. In fact, the present study provides only the boundary conditions on the flame front, considered as a surface of discontinuity moving in the turbulent flow. Moreover, when the flow inhomogeneities are too strong, the front is expected to possibly experience local quenching [8]. This mechanism which requires the study of higher orders in the expansion in powers of $\varepsilon$ is not addressed in the present study which can be used only for describing the dynamical behaviour of the flamelets far from the quenching limits. The results obtained here are also useful for describing the shape of the front in laminar flows when the corrugations have large amplitude as, for example, in the tip of a Bunsen flame or during the propagation in tubes.

The results obtained in this paper are very general; it turns out that only one scalar controls the local dynamics and the shape of the front i.e. the stretch of the surface experienced by the front during its motion in the gas flow. This quantity is proved to control the normal component of the front velocity relative to the flow. The relation between these two quantities is linear for small values of $\varepsilon$ and the corresponding coefficient depends on the detailed kinetics of the chemical reaction sustaining the heat release. Its expression is obtained here for a one step overall chemical 
reaction far from the stoichiometric composition and can be generalized to more realistic cases. The role of the flame stretch is more easily understood in the case of a planar flame stabilized in a stagnation point flow represented in figure 1 . The heat leaving the reaction zone by diffusion for preheating the incoming gas is equal to the heat released by the chemical reaction. The rate of this heat production is only controlled by the temperature of combustion resulting of the enthalpy balance. Inside the preheated zone, the reaction rate can be neglected and the convection balances the diffusion. Due to the flame stretch, transverse convection is added to the convection produced in the normal direction by the flame speed both of which take out the heat entering by diffusion from the reaction zone. Thus, for a given temperature of combustion, the flame speed is expected to be lowered by a positive stretch. But, the overall effect of flame stretching is more difficult to be anticipated because the temperature of combustion $T_{\mathrm{b}}$ depends on the difference between the heat diffusivity and the molecular diffusivity.

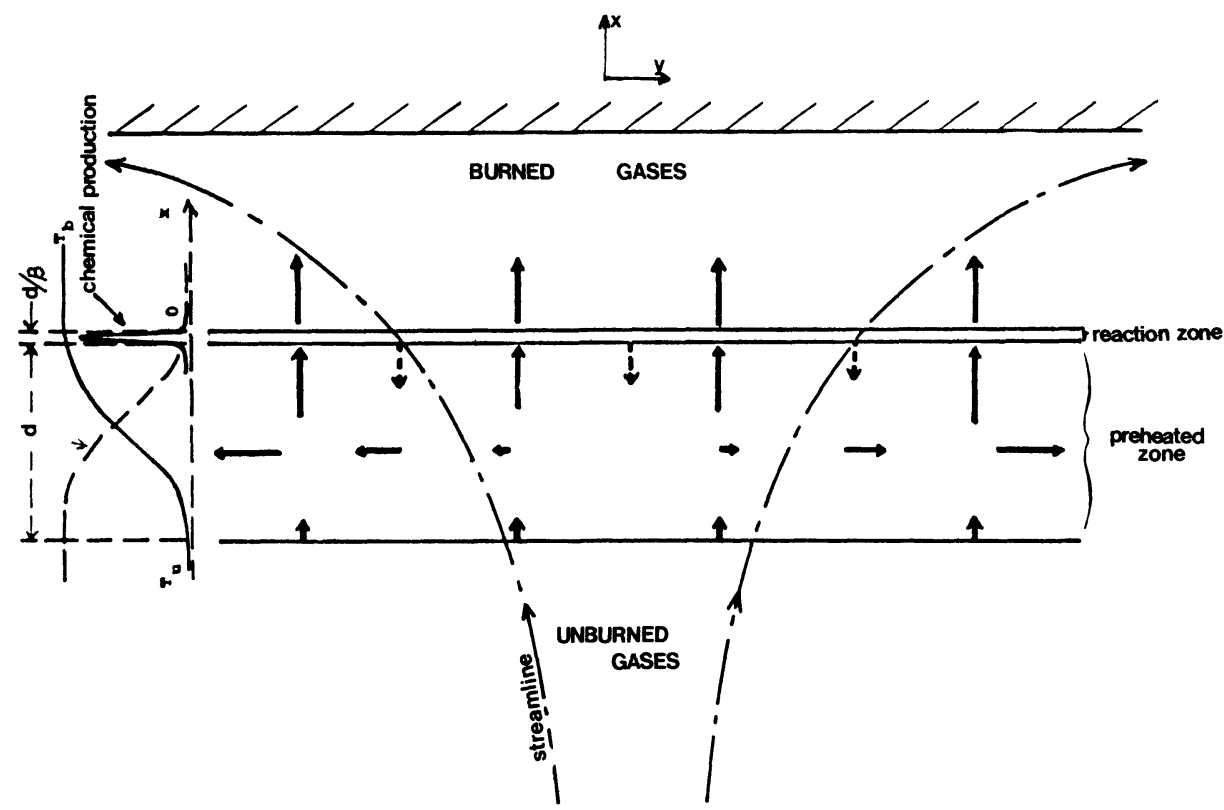

$\uparrow$ Longitudinal heat convection flux.

$\rightleftarrows \Rightarrow$ Transverse convective flux.

Diffusive heat flux.

Fig. 1. - Structure of a stretched planar flame stabilized in a stagnation point flow.

In fact the concept of flame stretch was first introduced a long time ago, in 1953, by Karlowitz [8] in a phenomenological description of the flame quenching in burners. But ten years later, Markstein [9], following the semi-analytical theory of Eckhauss [10], assumed that the relevant quantity controlling the flame speed was the curvature of the front relative to the " curvature " of the flow defined as the divergence of the unit flow velocity vector. The present analysis clarifies this aspect of the question and reconciles the two points of view of Karlowitz and Markstein. It is shown that the total stretch appearing in the final result can in fact, be expressed, in the limit $\varepsilon \rightarrow 0$, as the difference between the mean curvature of the front and a scalar built on the "rate of strain tensor " of the upstream flow and on the unit vector perpendicular to the front. This scalar differs from that introduced in the phenomenological approach of Markstein; 
it measures in fact the contribution of the flow inhomogeneity to the total stretch of the front. As for the curvature of the front, it can be interpreted as measuring the stretch produced by the natural motion of the front under a constant normal velocity equal to the laminar flame speed.

Furthermore, the time averaging of the non linear equation obtained for the local evolution of the front provides informations concerning the burning rate of a wrinkled flame stabilized in a turbulent flow. It is found that, even in the high intensity case, the turbulent flame speed is given by the laminar speed times the average ratio of the wrinkled flame area to the cross sectional area.

The problem will be formulated in the following section. The analysis appears in the subsequent section and can be omitted in a first reading. The reader is referred to references [2] and [6] for a detailed presentation of the two techniques used herein i.e. the asymptotic expansion for large values of the activation energy and the multiscale method based on $\varepsilon=d / \Lambda \ll 1$. Nevertheless this paper is self contained and all important steps are presented here even if they can be found elsewhere. The results are presented and discussed in the last section.

2. Formulation. - The flame model is the same as in the previous work [1,2]. Attention is restricted to exothermic reactions amenable to a one-step approximation, with the degree of progress describable in terms of the mass fraction $\psi_{A}$ of a single reactant, taken to be a limiting or deficient reactant in the sense that $\psi_{A}=0$ defines completion of the reaction. An Arrhenius rate is adopted such that as the reduced activation energy goes to infinity, the chemistry is confined to a fluctuating surface, termed the reaction zone whose position is employed to define the origin of a moving coordinate system [6]. With the density $\rho$ variable, the mass based thermal diffusivity $\rho D_{\text {th }}$ is assumed to be constant for simplicity. The effect of the temperature dependence of $\rho D_{\text {th }}$ have been studied in [11] for small amplitudes of the front corrugations and can easily be anticipated afterwards in the case of high amplitudes. The flame thickness $d=\rho D_{\mathrm{th}} / \rho_{\mathrm{u}} u_{\mathrm{L}}$ and the transit time $t_{\mathrm{L}}=d / u_{\mathrm{L}}$ are the units of length and time respectively. $u_{\mathrm{L}}$ is the laminar flame speed and the subscripts $u$ or $-\infty$ refer to the conditions in the fresh mixture. Let $U_{\mathrm{T}}=\left(U_{\mathrm{T}}, 0,0\right)$ and $\underline{U}_{-\infty}(X, Y, Z, T)=\left(U_{-\infty}, V_{-\infty}, W_{-\infty}\right)$ be the uniform and non-uniform parts of the upstream flow velocity in the nondimensional form respectively. $\underline{U}_{-\infty}$ is assumed to vary on nondimensional time and length scales of order $1 / \varepsilon ;(X, Y, Z, T)=(\varepsilon x, \varepsilon y, \varepsilon z, \varepsilon t)$ where $x, y$, $z, t$ are the nondimensional coordinates in the laboratory frame. The approximation $\varepsilon \ll 1$ means that the space scale of the upstream flow is larger than the flame thickness $\left(d \sim 10^{-2} \mathrm{~cm}\right)$. The scaling of the time is then given by the Taylor hypothesis. The turbulent flame speed $u_{\mathrm{T}}$ is assumed to be of the same order of magnitude as the laminar one $u_{\mathrm{L}}, U_{\mathrm{T}}=0(1)$ in the limit $\vec{\varepsilon} \rightarrow 0$. But, in contrast with [1], where the small amplitudes case was studied $\left(U_{-\infty}=0(\varepsilon)\right.$ ), we are looking here to the case of high turbulent intensity when $U_{-\infty}=0(1)$. Let the location of the flame sheet be defined by $x=\alpha(y, z, t)$ in the nondimensional variable. The order of magnitude of the amplitude $\alpha$ of the front corrugations can easily be evaluated by assuming that the normal burning velocity $u_{\mathrm{n}}$ is only weakly modified by the wrinkling, $\left(u_{\mathrm{n}} / u_{\mathrm{L}}\right)-1=0(\varepsilon)$ such a way that one must have $\partial \alpha / \partial t \sim U_{-\infty}$. This yields $\alpha=\frac{1}{\varepsilon} A(Y, Z, T)$ with $A=0(1)$ in the limit $\varepsilon \rightarrow 0$. The moving coordinates $\xi=x-\alpha, \eta=y, \Xi=z, \tau=t$ are introduced. $U_{\mathrm{T}}+\underline{U}_{-\infty}(X, Y, Z, T)$ is the flow velocity outside the flame thickness in the fresh mixture. But due to the heat release, the flow velocity must be modified inside the flame thickness on a length scale $d$. Let $\underline{U}=(U, V, W)$ be the components of the velocity in the $(x, y, z)$ coordinate system. Let $r=\rho / \rho_{\mathrm{u}}$ be the density ratio, $\underline{V}=(V, W)$ represent the transverse velocity, $\underline{\nabla}$ and $\underline{\hat{v}}$ denote the gradient involving differentiation with respect to $x, y, z$ and $\varepsilon \xi, \varepsilon \eta, \varepsilon \Xi$ respectively.

$$
S=r(U-\partial A / \partial T-\underline{V} \cdot \underline{\nabla} A)
$$

identify the nondimensional longitudinal mass flux (reduced by $\rho_{\mathrm{u}} u_{\mathrm{L}}$ ) in the moving frame. In 
the moving coordinate system $(\xi, \eta, \Xi)$, the conservation equation for mass, (2), and for longitudinal and transverse momentum, (3), (4), read

$$
\begin{aligned}
\partial S / \partial \xi & =-\varepsilon(\partial r / \partial T+\underline{\hat{\nabla}} \cdot(r \underline{V})) \\
S \partial U / \partial \xi+\partial P / \partial \xi & =-\varepsilon(r \partial U / \partial T+r \underline{V} \cdot \hat{\hat{v}} U) \\
S \partial \underline{V} / \partial \xi-(\underline{\hat{\nabla}} A) \partial P / \partial \xi & =-\varepsilon(r \partial \underline{V} / \partial T+r \underline{V} \cdot \underline{\hat{\nabla}} V+\underline{\hat{\nabla}} P)
\end{aligned}
$$

$P$ is the nondimensional pressure reduced by $\rho_{\mathrm{u}} u_{\mathrm{L}}^{2}$. The viscous forces have not been written for simplicity because, as it was shown in the previous works for small amplitude corrugations $[1,2,11]$, it is anticipated that the overall effect is neutral in such a way that the Prandtl numbers does not appear in the final result for the dynamical properties of the flame fronts.

In the approximation of vanishingly small Mach number, the density $r$ is related to the temperature through the perfect gas law by

$$
r=(1+\theta \gamma /(1-\gamma))^{-1}
$$

$\theta=\left(T-T_{\mathrm{u}}\right) /\left(T_{\mathrm{f}}-T_{\mathrm{u}}\right)$ and $\gamma=1-\left(\rho_{\mathrm{f}} / \rho_{\mathrm{u}}\right),(0<\theta, \gamma<1)$, are the nondimensional temperature and the expansion parameter respectively. The subscript $f$ refers to the conditions in the burned gases of the planar and adiabatic case. The temperature $\theta$ and the nondimensional mass fraction $\psi(\psi=1$ in the fresh mixture) of the limiting component obey the equations of energy and species conservation. These two equations are coupled by a production term with a temperature dependence given by an Arrhenius law and characterized by an activation energy $E$. When the reduced activation energy $\beta=\gamma \frac{E}{k T_{\mathrm{f}}}$ goes to infinity, the production term is confined to the surface $\xi=0$ and can be replaced by the following jump conditions resulting of the quasi steady and planar character of the reaction zone $[1,2,6]$ :

$$
\begin{gathered}
\partial \theta /\left.\partial \xi\right|_{\xi=0_{-}}=\left(1+|\hat{\nabla} A|^{2}\right)^{-1 / 2} \exp \left\{\frac{\beta}{2}\left(\theta_{\xi=0}-1\right)\right\} \\
{\left[L_{\mathrm{e}}^{-1} \partial \psi / \partial \xi+\partial \theta / \partial \xi\right]_{\xi=0-}^{\xi=00_{-}}=0,}
\end{gathered}
$$

(7) express the conservation of the enthalpy through the reaction zone and (6) states that the heat diffusion flux leaving the reaction zone along the normal direction toward the preheated zone is balanced by the chemical production of heat released inside the reaction zone. These relations are valid in the asymptotic limit $\beta \rightarrow \infty$ i.e. when the thickness of the reaction zone shrinks to zero, up to the order $1 / \beta$, [6]. (6) shows that the relative difference between the temperature of combustion $T_{\mathrm{b}}$ and the adiabatic flame temperature $T_{\mathrm{f}}$ must be of order $1 / \beta$ in the limit

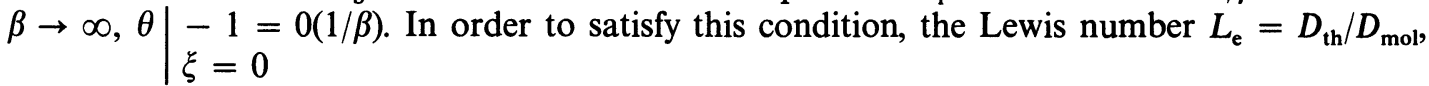
which is the ratio of the thermal diffusivity and of the molecular diffusivity, must be close to unity [6], $L_{\mathrm{e}}-1=0(1 / \beta)$,

$$
l=\beta\left(L_{\mathrm{e}}-1\right)=0(1) .
$$

When the viscous effects are overlooked, the jump conditions concerning the flow field are

$$
[U]_{0_{-}^{+}}^{0_{+}}=[\underline{V}]_{0_{-}^{+}}^{0_{+}}=[P]_{0_{-}}^{0_{+}} .
$$

Outside the thin reaction zone, the energy and species conservation yields,

$$
\begin{gathered}
\left\{r \frac{\partial}{\partial T}+r U \frac{\partial}{\partial x}+r \underline{V} \cdot \underline{\nabla}-\nabla^{2}\right\} \theta=0, \\
\left\{r \frac{\partial}{\partial T}+r U \frac{\partial}{\partial x}+r \underline{V} \cdot \underline{\nabla}-\frac{1}{L_{\mathrm{e}}} \nabla^{2}\right\} \psi=0 .
\end{gathered}
$$


When written in the moving coordinate system, these equations take the following form :

$$
\begin{aligned}
& L_{\mathrm{e}}^{-1}\left(1+|\hat{\nabla} A|^{2}\right) \partial^{2} \psi / \partial \xi^{2}-S \partial \psi / \partial \xi= \\
& \quad=+\varepsilon\left\{r \partial \psi / \partial T+r \underline{V} \cdot \underline{\hat{\nabla}} \psi+L_{\mathrm{e}}^{-1}\left(\hat{\nabla}^{2} A\right) \partial \psi / \partial \xi+2 L_{\mathrm{e}}^{-1} \partial(\underline{\hat{\nabla}} A \cdot \underline{\hat{\nabla}} \psi) / \partial \xi\right\}-\varepsilon^{2} L_{\mathrm{e}}^{-1} \hat{\nabla}^{2} \psi
\end{aligned}
$$

with the following boundary conditions, $\xi \rightarrow-\infty: \theta=0, \psi=1 ; \xi>0: \psi=0 ; \xi \rightarrow-\infty$ : $\theta=0$. A similar equation (10') holds for $\theta$ but where $L_{\mathrm{e}}$ is replaced by 1 . (10) emphasizes the order of magnitude in the limit $\varepsilon \rightarrow 0$ when the time derivative and the space variation in the transverse direction are anticipated to be of the same order of magnitude as for the upstream flow $U_{-\infty}$. The system (2)(3)(4)(5)(10) with the jump conditions (6)(7)(9) is to be solved in perturbation of $\varepsilon$ in order to obtain the equation for the evolution of the flame front. The two parameters of expansion $\beta^{-1}$ and $\varepsilon$ are independent, one comes from the chemistry and the other from the limited range of the wave lengths under investigation. The asymptotic limit $\beta \rightarrow+\infty$ is taken before the limit $\varepsilon \rightarrow 0$.

3. Analysis. - By identifying the contributions of the upstream flow (referred by the subscript $-\infty)$ and of the flame thickness (referred by ) respectively, the solutions of the equations (2), (3) and (4) can be written for $\xi<0$ in the following form :

$$
\begin{aligned}
S & =S_{-\infty}(A+\varepsilon \xi, \varepsilon \eta, \varepsilon \Xi, \varepsilon \tau)+\hat{S}(\xi, \varepsilon \eta, \varepsilon \Xi, \varepsilon \tau), \\
U & =U_{\mathrm{T}}+U_{-\infty}(A+\varepsilon \xi, \varepsilon \eta, \varepsilon \Xi, \varepsilon \tau)+\hat{U}(\xi, \varepsilon \eta, \varepsilon \Xi, \varepsilon \tau), \\
\frac{V}{P} & =\underline{V}_{-\infty}(A+\varepsilon \xi, \varepsilon \eta, \varepsilon \Xi, \varepsilon \tau)+\hat{V}(\xi, \varepsilon \eta, \varepsilon \Xi, \varepsilon \tau), \\
P & =P_{-\infty}(A+\varepsilon \xi, \varepsilon \eta, \varepsilon \Xi, \varepsilon \tau)+\hat{P}(\xi, \varepsilon \eta, \varepsilon \Xi, \varepsilon \tau) .
\end{aligned}
$$

Noticed that all the quantities with ^ vary as $\theta$ and $\psi$ on the length scale $d$ in the longitudinal direction and must go to zero when $\xi \rightarrow-\infty$ (upstream boundary of the flame thickness). Although the hydrodynamical effects described in $b$ ) of $\S 1$, are known to make $\underline{U}_{-\infty}$ to be a functional of the position of the front $A, \underline{U}_{-\infty}$ is considered here as a given quantity and only the flow modification $\hat{U}$ inside the flame is to be determined in the study of the flame structure. According to (5) one has $\lim _{\xi \rightarrow-\infty} r=1$ and the definition (1) provide the expression $S_{-\infty}$ :

$$
S_{-\infty}=U_{\mathrm{T}}+U_{-\infty}-\partial A / \partial T-\underline{V}_{-\infty} \cdot \underline{\nabla} A,
$$

in such a way that the normal burning velocity can be expressed as :

$$
\frac{u_{\mathrm{n}}}{u_{\mathrm{L}}}=\frac{\left.S_{-\infty}\right|_{x=A}}{\left(1+|\hat{\nabla} A|^{2}\right)^{1 / 2}} .
$$

This quantity is in fact the main unknown of the problem and may be considered as an " eigenquantity " determining the flame motion. $S_{-\infty}$ will be determined at the dominant order in the limit $\beta \rightarrow \infty$ and up to $0(\varepsilon)$ in the limit $\varepsilon \rightarrow 0$. Thus, the following expansions are introduced

$$
\begin{aligned}
&\left(S_{-\infty}, U_{-\infty}, \frac{V}{-\infty}\right)=\left(S_{-\infty, 0}, U_{-\infty, 0}, \underline{V}-\infty, 0\right)+\varepsilon\left(S_{-\infty, 1}, U_{-\infty, 1}, \underline{V}-\infty, 1\right)+0\left(\varepsilon^{2}\right), \\
&(\hat{S}, \hat{U}, \hat{P})=\left(\hat{S}_{0}, \hat{U}_{0}, \hat{P}_{0}\right)+\varepsilon\left(\hat{S}_{1}, \hat{U}_{1} \hat{P}_{1}\right)+0\left(\varepsilon^{2}\right) \\
& \underline{\hat{V}}=\underline{\hat{V}}_{0}+\varepsilon \underline{\hat{V}}_{1}+0\left(\varepsilon^{2}\right) \\
& \theta(\xi, \varepsilon \eta, \varepsilon \Xi, \varepsilon \tau)=\hat{\theta}_{0}+\varepsilon \hat{\theta}_{1}+0\left(\varepsilon^{2}\right), \\
& \psi(\xi, \varepsilon \eta, \varepsilon \Xi, \varepsilon \tau)=1-\hat{\psi}_{0}+\varepsilon \hat{\psi}_{1}+0\left(\varepsilon^{2}\right), \\
& A=A_{0}+\varepsilon A_{1}+0\left(\varepsilon^{2}\right) .
\end{aligned}
$$


At the zeroth order, $\varepsilon^{0},(2)$ implies $\partial \hat{S}_{0} / \partial \xi=0$ and then $\hat{S}_{0}=0$. (10) yields for $\xi<0$ :

$$
\begin{aligned}
& \hat{\psi}_{0}=\exp \left\{\xi L_{\mathrm{e}}\left(1+|\hat{\nabla} A|^{2}\right)^{-1} \cdot S_{-\infty}\right\} 0, \\
& \hat{\theta}_{0}=\hat{\theta}_{0}(\xi=0) \exp \left\{\xi\left(1+|\hat{\nabla} A|^{2}\right)^{-1} . S_{-\infty}\right\} 0 .
\end{aligned}
$$

Then the jump condition (7) implies $\hat{\theta}_{0}(\xi=0)=1+0(1 / \beta)$ and (6) yields :

$$
S_{-\infty, 0}=\left(1+|\hat{\nabla} A|^{2}\right)_{0}^{1 / 2} \text {. }
$$

Recalling (12), the result (15) means that, at the dominant order, $u_{\mathrm{n}} / u_{\mathrm{L}}=1$, the normal burning velocity is not modified. This is a trivial result because, in the limit $\varepsilon \rightarrow 0$, the effect of the wrinkling must effectively disappear. The following non trivial order will give the first correction. From (14) and (5) one obtains the expression for $r_{0}$ and then, (1), (3) and (4) provide the value of $\hat{U}_{0}, \hat{P}_{0}$ ?nd $\hat{V}_{0}$

$$
\begin{aligned}
\frac{1}{r_{0}}-1 & =\frac{\gamma}{1-\gamma} \exp \left\{\xi\left(1+|\nabla A|^{2}\right)_{0}^{-1 / 2}\right\} \\
\xi<0, \quad \hat{U}_{0} & =\left(\frac{1}{r_{0}}-1\right)\left(1+|\hat{\nabla} A|^{2}\right)_{0}^{-1 / 2}, \\
\hat{V}_{0} & =-\hat{U}_{0} \underline{\hat{\nabla}} A_{0},
\end{aligned}
$$

(16) corresponds to the usual deflection of the streamlines through the front produced by the modification of the normal component of the relative velocity.

Then, a straightforward integration of the mass balance (2) provides $\hat{S}_{1}(\xi)$ to give

$$
\hat{S}_{1}(\xi=0)=\left\{\frac{\partial}{\partial T}\left(1+|\hat{\nabla} A|^{2}\right)^{1 / 2}+\underline{\hat{\nabla}} \cdot\left(\left.\underline{V}_{-\infty}\right|_{x=A}\left(1+|\hat{\nabla} A|^{2}\right)^{1 / 2}\right)+\hat{\nabla}^{2} A\right\}_{0} \ln \left(\frac{1}{1-\gamma}\right) .
$$

In order to obtain the modification of the normal burning velocity given by $S_{-\infty, 1}$, it is necessary to compute the modification of the temperature of combustion $\hat{\theta}_{1}(\xi=0)$. This can be carried out by integrating from $\xi=-\infty$ to $\xi=0$ the order $\varepsilon$ of the sum of $(10)\left(10^{\prime}\right)$ and (2) times $(\theta+\psi-1)$ to give

$$
\hat{\theta}_{1}(\xi=0)=-\frac{\{\}_{0}}{\left(1+|\nabla A|^{2}\right)_{0}^{1 / 2}}\left(L_{\mathrm{e}}-1\right) \frac{1-\gamma}{\gamma} \int_{0}^{\gamma / 1-\gamma} \mathrm{d} x \frac{\ln (1+x)}{x},
$$

where \{\} is the same bracket as in (17). The derivation of (18) has required the use of the jump condition (7) with $\partial \theta /\left.\partial \xi\right|_{\xi=0_{+}}=0\left(\varepsilon^{2}\right)$ and the fact that, as $\left(L_{\mathrm{e}}-1\right), \hat{\theta}(\xi=0)$ is of order $\beta^{-1}$ in the limit $\beta \rightarrow \infty$. Moreover one can compute the modification of the heat flux entering the combustion zone by integrating from $\xi=-\infty$ to $\xi=0$ the difference between $\left(10^{\prime}\right)$ times $(1 / 1-\gamma)$ and $(2)$ times $r^{-1}=1+\theta(\gamma / 1-\gamma)$ to give at the order $\varepsilon$

$$
\left(\left.\left(1+|\hat{\nabla} A|^{2}\right) \frac{\partial \theta}{\partial \xi}\right|_{\xi=0-}\right)_{1}=S_{-\infty 1}(A, \varepsilon \eta, \varepsilon \Xi, \varepsilon \tau)+\frac{1}{\gamma} \hat{S}_{1}(\xi=0),
$$

where the expansion in power of $\varepsilon$ has been used for $S_{-\infty}(A+\varepsilon \xi)$. The final result is obtained by introducing (18) and (19) with (17) in the jump relation (6) expanded up to the order $\varepsilon$ to give

$$
S_{-\infty}(A)=\left(1+|\hat{\nabla} A|^{2}\right)^{1 / 2}-\varepsilon(\mathcal{L} / d)\{\}_{0}+0\left(\varepsilon^{2}\right) .
$$


The bracket \{\}$_{0}$ is the same as in $(17)$ and $(\mathcal{L} / d)$ is a non-dimensional coefficient given by

$$
\mathcal{L} / d=\frac{1}{\gamma} \ln \frac{1}{1-\gamma}+\frac{\beta\left(L_{\mathrm{e}}-1\right)}{2}\left(\frac{1-\gamma}{\gamma}\right) \int_{0}^{\gamma / 1-\gamma} \mathrm{d} x \frac{\ln (1+x)}{x},
$$

$\mathcal{L}$ as defined by (21), is a length that we will call "Markstein length ", in fact, such a length was introduced for the linear case in the phenomenological approach of Markstein [9]. According to (12) and (12'), the result (20), when expressed in terms of the normal burning speed $u_{n}$, takes the following form :

$$
\left(u_{\mathrm{n}} / u_{\mathrm{L}}-1\right)=-\varepsilon(\mathcal{L} / d)\{\}_{0}\left(1+|\nabla A|^{2}\right)_{0}^{-1 / 2}+0\left(\varepsilon^{2}\right) .
$$

4. Results and discussion. - Turbulent flame speed. - When the expression (12)-(12') of the normal burning velocity $u_{\mathrm{n}}$ introduced in (22), the final result provides a local equation for the evolution of the front, $x=\alpha(y, z, t)$ which takes the following dimensionalized form :

$$
\begin{aligned}
-u_{\mathrm{T}}-\left.u_{-\infty}\right|_{\alpha}+ & \partial \alpha / \partial t+\left.\underline{v}_{-\infty}\right|_{\alpha} \cdot \underline{\nabla} \alpha+u_{\mathrm{L}}(1+|\nabla \alpha|)^{1 / 2}= \\
& =\left\{\left\{\frac{\partial}{\partial t}\left(1+|\nabla \alpha|^{2}\right)^{1 / 2}+\underline{\nabla} \cdot\left(\left.\underline{v}_{-\infty}\right|_{\alpha}\left(1+|\nabla \alpha|^{2}\right)^{1 / 2}\right)+u_{\mathrm{L}} \nabla^{2} \alpha\right\}\right.
\end{aligned}
$$

with $\left(u_{-\infty}, \underline{v}_{-\infty}\right)=\left(u_{\mathrm{L}} U_{-\infty}, u_{\mathrm{L}} \underline{V}_{-\infty}\right)$ denoting the non uniform part of the upstream flow field.

When the random process is stationary as well as homogeneous in transverse directions on time average, the time average of local modifications to structure of laminar flamelets is zero and do not influence the turbulent flame speed. This result generalizes the one obtained for small amplitudes of the front corrugation [1]. Only the effect of an area increase associated with front wrinkling appears; the turbulent flame speed is equal to the laminar speed times the mean area increase :

$$
u_{\mathrm{T}}=u_{\mathrm{L}} \sqrt{1+|\nabla \alpha|^{2}}
$$

As it can be anticipated from [1], this result is limited only to the two first orders $\varepsilon^{0}$ and $\varepsilon^{1}$ of the expansion in powers of $\varepsilon$.

Flame stretch. - Following Karlowitz [8], one may seek to interprete the final result in terms of flame stretch. In dimensionalized form, (22) reads :

$$
\left(u_{\mathrm{n}} / u_{\mathrm{L}}-1\right)=-\frac{u_{\mathrm{L}}^{-1} \mathcal{L}\left\{\frac{\partial}{\partial t}\left(1+|\nabla \alpha|^{2}\right)^{1 / 2}+\underline{\nabla} \cdot\left(\underline{v}-\left.\infty\right|_{\alpha}\left(1+|\nabla \alpha|^{2}\right)^{1 / 2}\right)+u_{\mathrm{L}} \nabla^{2} \alpha\right\}}{\left(1+|\nabla \alpha|^{2}\right)^{1 / 2}}+0\left(\varepsilon^{2}\right) .
$$

The stretch can be defined precisely as the time derivative of the logarithm of an elementary area $\sigma$ of the flame front the points of which move in the flame surface with a tangential velocity equal to the tangential component of the gas velocity immediately ahead of the surface [17]. The normal velocity is taken to be the normal velocity of the front in order that the points of $\sigma$ remain 
in the flame surface. The mathematical expression of such a stretch has been given by Buckmaster [12] :

$$
\left(\frac{1}{\sigma} \frac{\mathrm{d} \sigma}{\mathrm{d} t}\right)_{\text {tot }}=\underline{\nabla}\left(\left.v\right|_{\alpha}\right)+\underline{\nabla} \cdot\left(\frac{u_{\mathrm{n}}}{\left(1+|\nabla \alpha|^{2}\right)^{1 / 2}} \underline{\nabla} \alpha\right)+\frac{D}{D t} \ln \left(1+|\nabla \alpha|^{2}\right)^{1 / 2},
$$

with

$$
\frac{D}{D t}=\frac{\partial}{\partial t}+\left.\underline{v}\right|_{\alpha} \cdot \underline{\nabla}+\frac{u_{\mathrm{n}}}{\left(1+|\nabla \alpha|^{2}\right)^{1 / 2}}(\underline{\nabla} \alpha) \cdot \underline{\nabla}
$$

it is worthwhile emphasizing that the local quantity defined by $(25)$ is representative of the total stretch experienced by a surface moving in the flowfield $(u, v)$ with a motion characterized by a normal velocity relative to the gas equal to $u_{\mathrm{n}}$. This total stretch is not only a characteristic of the flow $(u, \underline{v})$ but depends also on $u_{\mathrm{n}}$. It is clear from $(25)$ that $t_{\mathrm{L}}\left(\frac{1}{\sigma} \frac{\mathrm{d} \sigma}{\mathrm{d} t}\right)_{\text {tot }}=0(\varepsilon)$, thus the dominant order in the limit $\varepsilon \rightarrow 0$ of the total stretch is obtained by introducing in (25) the dominant order of $u_{\mathrm{n}}$ given by (15), $u_{\mathrm{n}}=u_{\mathrm{L}}(1+0(\varepsilon))$. By comparison with (24) we obtain

$$
\left(u_{\mathrm{n}} / u_{\mathrm{L}}-1\right)=-\frac{\mathfrak{L}}{u_{\mathrm{L}}}\left(\frac{1}{\sigma} \frac{\mathrm{d} \sigma}{\mathrm{d} t}\right)_{\text {tot }}+0\left(\varepsilon^{2}\right) .
$$

Thus, as anticipated by Karlowitz [8], (26) expresses that, at the dominant non trivial order in $\varepsilon$, the total stretch is the only scalar controlling the normal burning velocity. This result is not in complete agreement with the conclusion of the analysis by Buckmaster [12] who questioned the usefulness of a criterion based only on the stretch defined by (25). In fact, following the previous work of Sivashinsky [13], Buckmaster [12] used the "slow varying flame model " defined by $\beta \rightarrow \infty, 1-L_{\mathrm{e}}=0(1)$ and $\varepsilon=1 / \beta$. In this way instead of (25), the " volumial " stretch is found to be the scalar controlling $u_{\mathrm{n}}$ in reference [12]. This quantity is in fact expressed as the sum of $\left(\frac{1}{\sigma} \frac{\mathrm{d} \sigma}{\mathrm{d} t}\right)_{\text {tot }}$ and the time derivative of the logarithm of the local flame thickness. Furthermore, the expression obtained in reference [12] for the coefficient $\mathcal{L} / d$ contains only the second part of the complete formula (21) the first part of which has been proved recently to be essential in the stability analysis of planar fronts [1,2]. As was shown in reference [14], the joint use of $1-L_{e}=0(1)$ and of $\varepsilon=1 / \beta$ lead, even in the planar case, to time derivative terms which, like the additional terms appearing in reference [12], are not relevant. As it has been shown in the stability analysis of the thermal diffusive model (see for example ref. [6]) the domain of stability is characterized by $1-L_{\mathrm{e}}=0(1 / \beta)$ and thus, the assumption $1 / L_{\mathrm{e}}=0(1)$ with $\frac{\partial}{\partial t}=0(\beta)$ leads to results including unsteady effects associated with planar fronts instabilities too for away from the stability limits for concerning usual premixed flames.

By using the two independent limits $\beta \rightarrow \infty$ and $\varepsilon \rightarrow 0$, the present analytical study not only confirms the importance of the flame stretch concept anticipated previously by phenomenological considerations but also provides the precise formulation of its influence on dynamics of the flame front. For usual values of the gas expansion $(\gamma>0.8)$ and of the diffusivities involved in the traditional hydrocarbon-air mixtures, (21) predicts, in contrast to the thermal diffusive approximation characterized by $\gamma=0$, a positive value of $\mathcal{L} / d$ for any composition with a possible exception for hydrogen flames [2]. Thus, in agreement with the experimental data, a positive stretch is predicted to produce a decrease of the normal burning velocity $u_{\mathrm{n}}$. But the value of $\mathcal{L} / d$ depends on the kinetics of the reaction and is also sensitive to the heat losses [15]. For each particular case, 
the expression of $\mathcal{L} / d$ can be easily obtained by using the linear approximation $[11,15]$. The formula (21) is valid only for adiabatic flames amenable to a one-step kinetics approximation with mass based thermal diffusivities assumed to be constant. But the results (23) and (26) are general and can be used for every premixed flames.

Front curvature. - It is worthwhile noticing that the early semi-analytical linear theory of Eckhauss [10] showed that the flame velocity is influenced by the joint effects of the front shape and of the flow inhomogeneities. An extension to the nonlinear case has been proposed by Markstein [9] who assumed that the relevant quantity could be the difference of the mean curvature of the front $\left(\frac{1}{R}=\frac{1}{R_{1}}+\frac{1}{R_{2}}\right.$ defined to be positive when the front is convex toward the burnt mixture) and of the " curvature of the upstream flow » defined as the divergence of a unit vector in the direction of the flow. Such an expression matches effectively the result (24) in the linear approximation,

$$
\left(u_{\mathrm{n}} / u_{\mathrm{L}}-1\right) \simeq-\mathcal{L}\left\{u_{\mathrm{L}}^{-1} \underline{\nabla} \cdot \underline{v}_{-\infty}+\nabla^{2} \alpha\right\}
$$

in this case, $1 / R \simeq-\nabla^{2} \alpha$ and $-\underline{\nabla} \cdot v_{-\infty}$ is the linear approximation of the curvature of the flow as defined by Markstein.

In fact the exact result (24) can easily be expressed in terms of $1 / R$ to give :

$$
\left(u_{\mathbf{n}} / u_{\mathrm{L}}-1\right)=+\mathcal{L}\left\{\frac{1}{R}+\frac{1}{u_{\mathrm{L}}} \mathbf{n} \cdot \nabla \mathbf{u}_{-\infty} \cdot \mathbf{n}\right\}+0\left(\varepsilon^{2}\right)
$$

where $\mathbf{n}$ is the unit vector normal to the front and $\mathbf{\nabla \mathbf { u } _ { - \infty }}$ is the " rate of strain tensor " of the upstream flow, $u_{-\infty}=\left(u_{-\infty}, v_{-\infty}\right)$, evaluated at the flame position. The second term of the r.h.s. of (28) is different from the quantity proposed by Markstein and can receive a very simple physical interpretation. The quantity $\underline{\nabla} \cdot \underline{u}_{-\infty}-\mathbf{n} \cdot \nabla \mathbf{u}_{-\infty} \cdot \mathbf{n}$ represents the relative growth rate, $\left(\frac{1}{\sigma} \frac{\mathrm{d} \sigma}{\mathrm{d} t}\right)$, of an infinitesimal area $\sigma$ of the surface perpendicular to $\mathrm{n}$ and convected by the flow $\underline{u}_{-\infty}$. Thus, as $\underline{\nabla} \cdot \underline{u}_{-\infty}=0,(28)$ can be written as :

$$
\left(u_{\mathrm{n}} / u_{\mathrm{L}}-1\right)=\mathfrak{L} u_{\mathrm{L}}^{-1}\left\{\frac{u_{\mathrm{L}}}{R}-\left(\frac{1}{\sigma} \frac{\mathrm{d} \sigma}{\mathrm{d} t}\right)_{\mathrm{conv}}\right\}+0\left(\varepsilon^{2}\right)
$$

$\left(\frac{1}{\sigma} \frac{\mathrm{d} \sigma}{\mathrm{d} t}\right)_{\text {conv }}$ is the stretch that the front would have experienced if it was only convected by the upstream flow $u_{-\infty}$. Thus the two different forms (26) and (29) of the same final result can be easily understood by noticing that $-\frac{u_{\mathrm{L}}}{R}$ is the stretch, $\left(\frac{1}{\sigma} \frac{\mathrm{d} \sigma}{\mathrm{d} t}\right)_{\mathrm{n}}$, that the front would have experienced if it moved in a upstream quiescent medium with a normal burning velocity equal to $u_{\mathrm{L}}$ : $\left(\frac{1}{\sigma} \frac{\mathrm{d} \sigma}{\mathrm{d} t}\right)_{\mathrm{tot}}=\left(\frac{1}{\sigma} \frac{\mathrm{d} \sigma}{\mathrm{d} t}\right)_{\mathrm{n}}+\left(\frac{1}{\sigma} \frac{\mathrm{d} \sigma}{\mathrm{d} t}\right)_{\text {conv }}$.

The main interest of the forms (28) and (29) is to separate in the total stretch, the contribution of the geometry of the front from the contribution of the inhomogeneities of the upstream flow.

Burned gases. - It is worthwhile to underline that we have chosen in this paper to express the final results in terms of the characteristics of the upstream flow $\underline{u}_{-\infty}$ just ahead of the front. 
It is easy, by using (9) (16) and (17) to express the result in term of the downstream flow $\underline{u}_{+\infty}=$ $\left(u_{+\infty}, \underline{v}_{+\infty}\right)$ just behind the reaction zone :

$$
\begin{aligned}
-u_{\mathrm{T}}^{\mathrm{b}}-\left.u_{+\infty}\right|_{\alpha}+\partial \alpha / \partial t & +\left.\underline{v}_{+\infty}\right|_{\alpha} \cdot \underline{\nabla} \alpha+u_{\mathrm{L}}^{\mathrm{b}}\left(1+|\nabla \alpha|^{2}\right)^{1 / 2}= \\
& =\mathcal{L}^{\mathrm{b}}\left\{\frac{\partial}{\partial t}\left(1+|\nabla \alpha|^{2}\right)^{1 / 2}+\underline{\nabla} \cdot\left(\left.\underline{v}_{+\infty}\right|_{\alpha}\left(1+|\nabla \alpha|^{2}\right)^{1 / 2}\right)+u_{\mathrm{L}}^{\mathrm{b}} \nabla^{2} \alpha\right\}
\end{aligned}
$$

with

$$
\mathcal{L}^{\mathrm{b}} / d=\frac{1}{\gamma}\left\{\ln \frac{1}{1-\gamma}+\frac{\beta\left(L_{\mathrm{e}}-1\right)}{2} \int_{0}^{\gamma / 1-\gamma} \mathrm{d} x \frac{\ln (1+x)}{x}\right\}
$$

with $u_{\mathrm{L}}^{\mathrm{b}} \equiv \frac{1}{1-\gamma} u_{\mathrm{L}}$ and $u_{\mathrm{T}}^{\mathrm{b}}$ denoting the velocity of the burned gas in the laminar and turbulent cases respectively. A similar relationship was deduced for the spherical geometry by Frankel and Sivashinsky [16] who noticed that contrarily to the value (21) of $\mathcal{L} / d$ obtained by Clavin and Williams [1], $\mathfrak{L}^{\mathbf{b}} / d$ given by (31) can easily change sign for the usual hydrocarbon-air flames. When expressed in terms of the normal burning velocity but relative to the burned gases, $u_{\mathrm{n}}^{\mathrm{b}}=\left\{u_{\mathrm{T}}^{\mathrm{b}}+\right.$ $\left.\left.u_{+\infty}\right|_{\alpha}-\partial \alpha / \partial t-\underline{v}_{+\infty} l_{\alpha} \cdot \underline{\nabla} \alpha\right\}\left(1+|\nabla \alpha|^{2}\right)^{-1 / 2}$ the result (30) can be written in a similar form to $(26)$ :

$$
\left(u_{\mathrm{n}}^{\mathrm{b}} / u_{\mathrm{L}}^{\mathrm{b}}-1\right)=-\frac{\mathcal{L}^{\mathrm{b}}}{u_{\mathrm{L}}^{\mathrm{b}}}\left(\frac{1}{\sigma} \frac{\mathrm{d} \sigma}{\mathrm{d} t}\right)_{\text {tot }}+0\left(\varepsilon^{2}\right)
$$

Thus a positive flame stretch can produce only a decrease of the normal burning velocity relative to the fresh mixture but can produce an increase of the normal burning velocity relative to the burned gases. This is possible because the modification of the flame structure by the front wrinkling involves a change of the normal mass flux, though the flame thickness. This result is very spectacular in spherical flame propagation in quiescent medium studied in reference [16]; in this case, the corresponding modification is due to unsteady effects. Each of the two forms (30)(32) and (25)-(27) can be useful depending on the configuration. For converging spherical flames, the fresh mixture is at rest, $\underline{u}_{-\infty}=0$, and (23)-(27) provide directly the front evolution. For diverging flames when the burned gases are at rest, (30)-(32) give directly the solution. In these cases, the difference between $\mathcal{L}$ and $\mathfrak{L}^{b}$ describes the difference in the propagation between a converging and a diverging flame. In the spherical steady case, one has $\frac{u_{\mathrm{L}}}{R}=\left(\frac{1}{\sigma} \frac{\mathrm{d} \sigma}{\mathrm{d} t}\right)_{\text {conv }}$ and thus, $\left(\frac{1}{\sigma} \frac{\mathrm{d} \sigma}{\mathrm{d} t}\right)_{\text {tot }}=0$. The two forms (27) and (32) show that there is no modification of the normal burning velocity in this [18] case. When, as for idealized Bunsen flames, the upstream flow can be considered as uniform $\underline{u}_{-\infty}=0,(23)$ gives directly the shape of the front. But in most of the cases, as for the propagation in tubes or for the turbulent wrinkled flames, $u_{+\infty}$ and $u_{-\infty}$ are unknown; then the complete solution of the problem requires the solution of the incompressible fluid mechanics problem ahead and behind the front. The relations (23) and (30) have to be considered as two of the boundaries conditions necessary to solve this difficult problem. The other jump conditions concerning the flow velocity and the pressure can be easily computed as in the reference [2]. 


\section{References}

[1] Clavin, P. and Williams, F. A., J. Fluid. Mech. 116 (1982) 251.

[2] Pelce, P. and Clavin, P., J. Fluid. Mech. 124 (1982) 219.

[3] Darrieus, G., 6th International Congress of Appl. Mech. (Paris) 1945.

[4] Landau, L., Acta Physicochim. URSS 19 (1944) 17.

[5] Barenblatt, G. I., Zeldovich, Y. and Istratov, A. G., Prikl. Mekh. Tekh. Fiz. 2 (1962) 21.

[6] Joulin, G. and Clavin, P., J. Comb. and Flame 35 (1979) 139.

[7] Bush, W. B. and Fendell, F. E., Comb. Sci. Technol. 1 (1970) 421.

[8] KaRlowitz, B. et al., IV Symposium on Combustion (1953) 613.

[9] Markstein, G. H., Non steady flame propagation (Pergamon Press) 1964.

[10] ECKhauss, W., J. Fluid. Mech. 10 (1961) 80.

[11] Clavin, P. and Garcia, P., J. Méc., to appear in January 1983.

[12] BuCKMaster, J., Acta Astronautica 6 (1979) 741.

[13] Sivashinsky, G. I., Acta Astronautica 3 (1976) 889.

[14] Joulin, G., Existence, stabilité et structuration des flammes prémélangées, Thèse, Poitiers (1979).

[15] Clavin, P. and Nicoli, C., Comb. and Flame, submitted (1982).

[16] Frankel, M. L. and Sivashinsky, G. I., J. Comb. Sci. Tech. submitted (1982).

[17] Williams, F. A., Agard Conference Proceedings no 164, ed. M. Barrere 1975.

[18] Deshaies, B., Joulin, G., Clavin, P., J. Méc. 20, no 4 (1981) 691. 